\title{
Patient's satisfaction after 2-piece inflatable penile prosthesis implantation: An Italian multicentric study
}

\author{
Giorgio Gentile ${ }^{1-2}$, Alessandro Franceschelli ${ }^{1}$, Paolo Massenio ${ }^{3}$, Agostino Tuccio ${ }^{4}$, \\ Andrea Cocci ${ }^{4}$, Lucia Divenuto ${ }^{5}$, Daniele Romagnoli ${ }^{2}$, Alessandro Natali ${ }^{4}$, Antonio Vitarelli ${ }^{5}$, \\ Luigi Cormio $^{3}$, Fulvio Colombo ${ }^{1}$ \\ ${ }^{1}$ Andrology-Unit, University Hospital S.Orsola-Malpighi, Bologna, Italy; \\ ${ }^{2}$ Department of Urology, University of Bologna, Bologna, Italy; \\ ${ }^{3}$ Department of Urology and kidney transplant Center, University of Foggia, Italy; \\ ${ }^{4}$ Department of Urology and Oncology, AOU Careggi, University of Florence, Italy; \\ ${ }^{5}$ Department of Urology, University of Bari “Aldo Moro", Italy.
}

\begin{abstract}
Summary Introduction: Penile prosthesis implant represents a valuable solution for pts with severe erectile dysfunction (ED), non-responders to medical management. The aim of our study was to evaluate the satisfaction of patients (pts) after 2-pieces inflatable penile prosthesis (IPP).

Aim of the study: to evaluate safety, reliability and post-operative patient's satisfaction after implantation of two-pieces IPP. Materials and Methods: This retrospective multicentric analysis concerns a group of 42 patients undergone 2-pieces IPP implantation from November 2005 to November 2013, in four Centers of proven experience. As a first step, a detailed review of all clinical reports was performed. Secondly, every patient was asked to fill the Erectile Dysfunction Inventory of Treatment Satisfaction (EDITS) specifically modified, in order to assess their own satisfaction after surgery and, its impact on patient's quality of sexual life.

Results: 42 pts were evaluated (AMS-Ambicor: 28; ColoplastExcell: 14); mean age, at time of operation: 60,7 years; mean follow up: 27,6 months; etiology of ED: vascular 23,8\%, diabetes $19 \%$, La Peyronie D. $7,1 \%$, consequence of radical prostatectomy $31 \%$, consequence of other pelvic surgery $11,9 \%$, spinal trauma $7 \%$. Mean operative time: $117 \pm 58 \mathrm{~min}$, mean postoperative hospital stay $3 \pm 1,6$ days.

Post operative short-term complications: 4 pts (9,5\%). Post operative long-term complications: 4 pts $(9,5 \%)$.

Long-term functional results (Questionnaire): $71 \%$ of pts (30) reported regular use of the prosthesis, at least 1 time/week, the satisfaction was good in $42 \%$ of pts (18), quite good in $33,3 \%$ (14), quite bad in 2,4\% (1), very bad in 7,1\% (3), 6 pts (14,4\%) didn't answer.

Conclusions: 2 pieces IPP appears to be associated with a low complication rate and good satisfaction of pts especially in the elderly. It also assures satisfactory rates of aesthetics and functional results.
\end{abstract}

KEY WORDS: Erectyle dysfunction; Patient satisfaction.

Submitted 5 February 2016; Accepted 15 February 2016

\section{INTRODUCTION}

Erectile Dysfunction (ED) is defined as "the persistent inability to attain and maintain an erection sufficient to permit satisfactory sexual performance" (1). The prevalence of this condition is about $19 \%$, and its medical treatment is based upon different options, ranging from oral administration to intracavernousal injections (2).

In patients (pts) not responsive to medical therapies, Penile Prosthesis Implant is the only chance (3).

Since their first appearance in 1970, penile prosthetic devices have progressively improved, thus becoming not only more reliable but also easier to be implanted and to be used (4). The 2-piece IPP is often considered a "second option" for these pts, although there are only few studies focused on their impact in patients' quality of life (5-9).

\section{Alms}

The aim of this study was to evaluate the clinical safety and reliability of two-pieces IPP implantation, and the level of acceptance and satisfaction in operated pts under different points of view: functional, sexual, behavioral and personal.

\section{Materials AND MEthods}

This paper reports a retrospective multicentric study regarding 42 patients undergone 2-pieces IPP from November 2005 to November 2013, in four different Centers of proven experience. Patients' age, cause of ED and possible cardiovascular risk factors were collected. Indication for surgery was given in case of severe organic ED refractory to any medical treatment.

Each patient received a detailed explanation about any possible risk related to the procedure, and a specific informed consent was obtained. Exclusion criteria were psychiatric illness, genital or systemic infections and any comorbidity affecting wound healing.

AMS Ambicor device was implanted in 29 cases (69\%), since a Coloplast Excel model was chosen in the remaining 13 (31\%). In all pts a peno-scrotal approach was applied. A detailed review of all clinical reports was performed and data were collected.

Each patient was then contacted by phone and asked to return to the referring hospital, for long-term clinical examination. In this occasion, every patient was asked to fill a specific questionnaire, a modified version of the val- 
idated questionnaire "Erectile Dysfunction Inventory of Treatment Satisfaction (EDITS)" (5-6), probing on four fundamental items: frequency of use of the implant, its impact on patient's quality of sexual life, personal evaluation of the outcomes of the operation, from both the functional and aesthetic point of view. Reponses were classified according to a five-point scale, in most cases ranging from "extremely satisfied (1)" to "totally unsatisfied (5)". Each interview was conducted by the same physician (one for each Institution). During the interview, the patient and his partner were placed in different areas.

\section{Results}

A total of 42 pts were implanted using 2-pieces IPP between November 2005 and November 2013. Mean age at the time of surgery was $61 \pm 10$, ranging from 33 to $80.50 \%$ of patients were smokers, $30 \%$ were affected by hypertension, 19\% had Type 2 Diabetes Mellitus (in effective pharmacological treatment) and 1\% had dyslipidemia (Table 1).

None of the patients had been previously implanted. Etiology of ED was: radical prostatectomy (33\%), vascular abnormalities (24\%), Diabetes mellitus (19\%), other pelvic surgery (12\%), Peyronie's disease (7\%), spinal trauma (5\%) Figure 1.

In 29 cases (69\%) the AMS Ambicor device was implanted, since a Coloplast Excel model was placed in the remaining 13 (31\%). Mean operative time recorded was of $117 \pm 58$ minutes, no intra-operatory complications had been reported. Antibiotic prophylaxis was administered in the operatory room, and each patient (100\%) was given systemic therapy with third generation cephalosporines (cephuroxime) until the hospital discharge. Mean hospital stay was $3 \pm 1.6$ days.

During hospitalization one patient presented hyperpyrexia, while two required prolonged analgesic therapy for post-operative pain. No blood transfusion was required, and there was no need of surgical revision in any case. Considering long term complications, two patients had prosthesis extrusion due to infection, one patients complained persistent loss of glans sensitivity, one patient presented prosthetic malfunction. In the remaining 37 cases (88\%), no late onset complication was documented. Mean follow up time was 27 months. At the follow up no patient was lost: 31 (73\%) patients were found to routinely use the prosthesis, 6 (14\%) didn't have a partner to use it with, 3 (7\%) lost interest in using (two of them because affected by oncological disease, the remaining one because of poor manageability of the implant), and two (4.8\%) reported pain at the activation of implant. When asked about the frequency of sexual activity, the response was 2 intercourse per week, average. Analyzing overall patients' satisfaction following the operation, $42 \%$ were extremely satisfied, 33\% referred to be almost satisfied, the remaining 25\% were substantially indifferent to the result. There were no case of total unsatisfaction. 29 pts (70\%) found almost full realization of their preoperative expectations, with $67 \%$ stated they were ready to use the device. In $90 \%$ of cases the operation granted the sexual self confidence the patients were searching for, and $73 \%$ of the partners
Table 1.

Characteristics of the population enrolled.

\begin{tabular}{|lc|}
\hline Age $($ mean \pm SD) (years) & $61 \pm 10$ \\
\hline Age range & $33-80$ \\
\hline Smokers & $50 \%$ \\
\hline Hypertension & $30 \%$ \\
\hline Dyslipidemia & $1 \%$ \\
\hline Diabetes & $19 \%$ \\
\hline
\end{tabular}

interviewed resulted to be fully satisfied. The device was found to be "user-friendly" in 76\% of the pts and only one patient defined the implant as "extremely difficult to use". 27 out of 42 pts (64\%) reported to be fully satisfied by the device, once activated, and the majority of pts reported fully satisfaction about the aesthetic appearance of the prosthesis when deactivated. Only three pts complained for the incomplete concealing of the prosthesis. If considering the filling system, only two pts reported a certain "delay" in the activation process.

One was not satisfied for the insufficient girth of the shaft, another one referred shortening of the penis and one was unsatisfied for incomplete penile rigidity with full-activated implant. Under the aestethical point of view the overall percentage of appreciation was 95\% (40 pts). Only two pts were dissatisfied due to the aspect of the penis when inflated (one for the girth, one for the lenght); moreover, one of them referred to be "not personally satisfied" by the overall appearance of the prosthesis. The length of the penis was reported to be increased in 13 of patients, reduced in 8 pts, and unmodified in the remaining 21 .

Figure 1.

Etiology of ED in the population of the study.

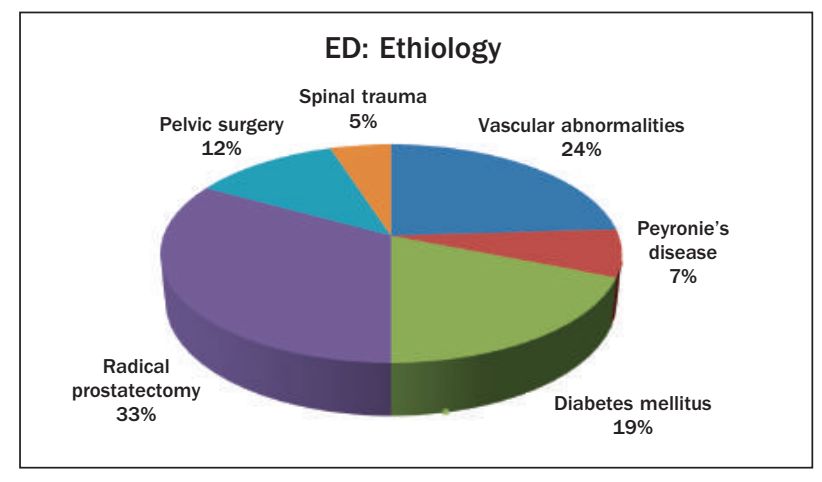

\section{Discussion}

IPP is the final option for the treatment of pts with ED (1). This is the only reliable solution for this population. According to our data the 2 piece IPP seems still to play a role in this field.

In our multicentric study no patient was lost during the follow up. The rate of satisfaction results consistent with data of literature (9). It is important to notice that, in our study, there were 15 patients (36\%) who were older than 65 years and all of them reported to be almost or very satisfied by the 2-piece IPP function (Figure 2), belonging the low rate of dissatisfaction only to patients younger than 65 
Figure 2.

Satisfaction rate (\%) in patients older than 65 years (15 pts).

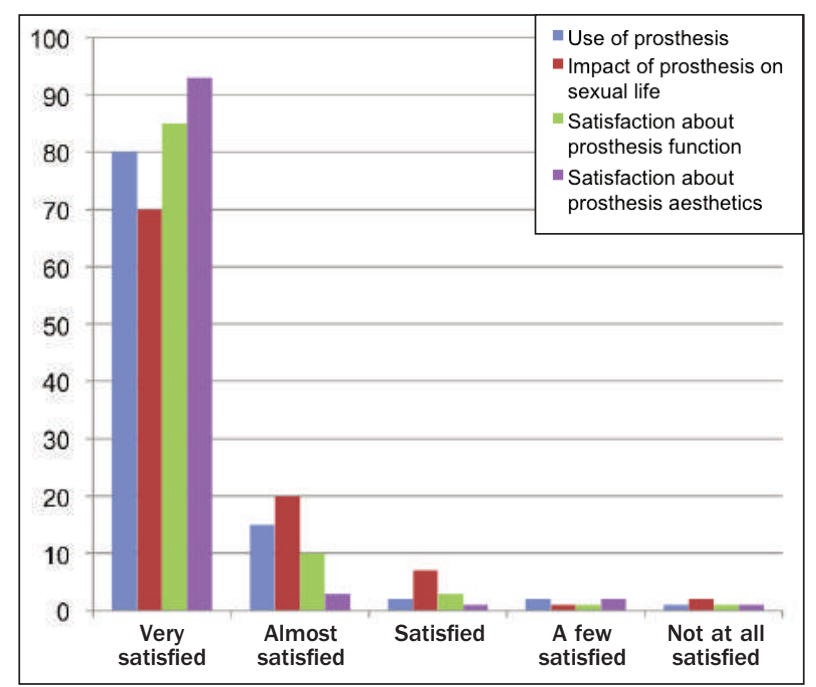

years (Figure 3). These data agree with many works about PPI (10-12). Explanation could be the lower expectations among elder pts. The low rate of complications observed (both early and late onset) confirms the reliability of the 2 piece-IPP.

Interestingly, this kind of implant seems to do not affect penile length (maybe due to the incomplete state of flaccidity of the penile shaft, typically provided by the 2pieces IPP when deactivated), thus dismantling one of those myths which prevents people affected by ED from undergoing IPP (11).

The aesthetical aspects of the prosthesis have substantially gained widespread appreciation, with acceptable concealing of the device.

\section{Conclusions}

IPP is a feasible solution to treat severe ED. The 2-pieces models are a valid option of choice, especially in the elder patient, and has low rates of intra and postoperative complications. It also offers satisfactory rates of aesthetics and functional results.

\section{REFERENCES}

1. Lue TF, Giuliano F, Montorsi F, et al. Summary of the recommendations on sexual dysfunctions in men. J Sex Med. 2004; 1:6-23.

2. Hatzimouratidis K, Amar E, Eardley I, et al. EAU Guidelines on male sexual dysfunction: erectile dysfunction and premature ejaculation, Eur Urol. 2010; 57:804-814.

3. Montague DK. Penile prosthesis implantation in the era of medical treatment for erectile dysfunction. Urol Clin North Am. 2011; $38: 217-25$.

4. Jarow JP. Risk factors for penile prosthesis infection, J Urol. 1996; 156:402-404.

5. Altof SE, Corty EW, Levine SB, et al. EDITS: development of questionnaires for evaluating satisfaction with treatments for ED. Urology. 1999; 53:793.

6. Lux M, Reyes-Vallejo L, Morgentaler A, Levine LA. Outcomes
Figure 3.

Satisfaction rate (\%) in patients younger than 65 years (27 pts).

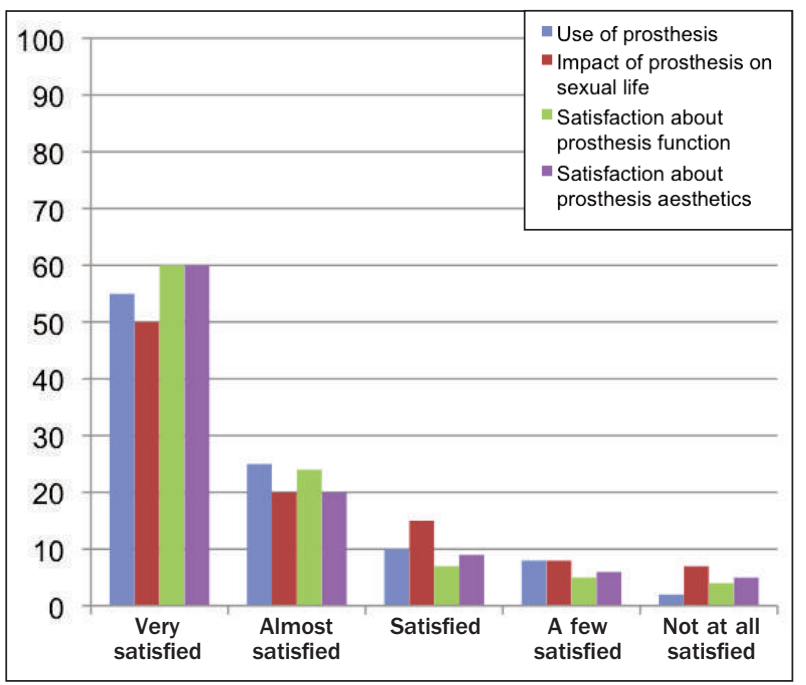

and satisfaction rates for the redesigned 2-piece prosthesis, J Urol. 2007; 177:262-266.

7. Levine LA, Estrada CR, Morgentaler A. Mechanical reliability and safety of, and patient satisfaction with the ambicor inflatable penile prosthesis: results of a 2 center study, J Urol. 2001; 166:932-937.

8. Mulhall JP, Ahmed A, Branch J, Parker M. Serial assessment of efficacy and satisfaction profiles following penile prosthesis surgery. J Urol. 2003; 169:1429-1433.

9. Bettocchi C, Palumbo F, Spilotros M, et al. Patient and partner satisfaction after AMS inflatable penile prosthesis implant. J Sex Med. 2010; 7:304-309.

10. Villareal HG, Jones L. Outcomes and satisfaction with the inflatable penile prosthesis in the elderly male. Adv Urol. 2012; 240963.

11. Al-Najar A, Naumann CM, Kaufmann S, et al. Should being aged over 70 years hinder penile prosthesis implantation? BJU Int. 2009; 104:834-837.

12. Lux M, Reyes-Vallejo L, Morgentaler A, Levine LA. Outcomes and satisfaction rates for the redesigned 2-piece prosthesis. J Urol. 2007; 177:262-6.

\section{Correspondence}

Giorgio Gentile, MD - dr.giorgio.gentile@gmail.com

Alessandro Franceschelli, MD - alessandro.franceschelli@aosp.bo.it Fulvio Colombo, MD - fulvio.colombo@aosp.bo.it

Andrology Unit, University Hospital S.Orsola-Malpighi, Bologna, Italy

Paolo Massenio, MD - p.massenio@alice.it

Luigi Cormio, MD - luigi.cormio@unifg.it

Department of Urology and kidney transplant Center,

University of Foggia, Foggia, Italy

Agostino Tuccio, MD - agostinotuccio@yahoo.it

Andrea Cocci, MD - cocci.andrea@gmail.com

Alessandro Natali, MD - anatali@dada.it

Department of Urology and Oncology, AOU Careggi, University of Florence Firenze, Italy

Lucia Divenuto, MD - luciadivenuto@hotmail.it

Antonio Vitarelli, MD - antoniovitarell@@otmail.com

Department of Urology, University of Bari "Aldo Moro", Bari, Italy

Daniele Romagnoli, MD - danieleromagnoli@hotmail.it

Department of Urology, University of Bologna, Bologna, Italy 